\title{
An invasive thymoma extending into the superior vena cava and right atrium
}

\author{
Tao Yang ${ }^{1}$, Ruting Hui ${ }^{2}$, Qingchen $\mathrm{Wu}^{1}$, Jie $\operatorname{Tian}^{1}$, Huanwen Chen ${ }^{1}$ \\ ${ }^{1}$ Department of Thoracic and Cardiovascular Surgery, The First Affiliated Hospital of Chongqing Medical University, Chongqing 400016, China; \\ ${ }^{2}$ Department of Rehabilitation, Chengdu First People's Hospital, Chengdu 610000, China \\ Correspondence to: Dr. Huanwen Chen. Department of Thoracic and Cardiovascular Surgery, The First Affiliated Hospital of Chongqing Medical \\ University, Youyi Road 1, Chongqing 400016, China. Email: coolstarchw9527@163.com.
}

\begin{abstract}
Invasive thymoma commonly infiltrates mediastinal structures; however, intracardiac extension from an intracaval growth is rare. An unusual case of an invasive thymoma protruding into the RA is here described. A left brachiocephalic vein (LBCV) tumor appeared through the thymic veins of a 50-year-old female patient. The patient underwent a combined resection of the invasive tumor and superior vena cava (SVC) and right atrium (RA) under cardiopulmonary bypass (CPB). Pathologic examination revealed this to be a WHO type B3 thymoma, Masaoka stage III. Following surgery, the patient's symptoms disappeared, and the quality of life improved. The patient was then introduced to radiotherapy and chemotherapy. This present case indicates that surgical treatment can create the opportunity for radiotherapy and chemotherapy, and is suitable and necessary for the treatment of invasive thymoma.
\end{abstract}

Keywords: Malignant thymoma; cardiopulmonary bypass (CPB); superior vena cava syndrome; intracardiac extension; superior vena cava resection

Submitted Mar 26, 2019. Accepted for publication Aug 12, 2019.

doi: $10.21037 /$ atm.2019.08.59

View this article at: http://dx.doi.org/10.21037/atm.2019.08.59

\section{Introduction}

Thymoma is considered to be a relatively rare type of tumor and has an incidence of only 0.15 cases per 100,000 in the global population (1). Invasive thymomas, accounting for approximately $30 \%$ of thymomas, may invade mediastinal organs such as the pleura and pericardium (2). Superior vena cava (SVC) syndrome associated with thymomas is rare (around $4 \%$ ). The most common cause is extrinsic compression (3). Invasive thymoma with the intravascular invasion of the SVC extending into the right atrium (RA) has only rarely been reported.

The present case describes a patient with an invasive thymoma extending into the SVC and RA and presents the clinical results that were observed following surgical treatment.

\section{Case presentation}

A 50-year-old female patient presented at the Department of Thoracic and Cardiovascular Surgery, The First Affiliated Hospital of Chongqing Medical University complained of swelling of the face and chest tightness having lasted 1 year. Upon physical examination, the patient's face was hydroncus, and her lips were cyanotic. Her blood pressure was $110 / 84 \mathrm{mmHg}$ (normal 90-140/90-60 $\mathrm{mmHg}$ ), her respiration was 18 times/min (normal 12-20 times/min), her heart rate was 85 beats $/ \mathrm{min}$ (normal $60-100$ beats $/ \mathrm{min}$ ), and her body temperature was $36.6^{\circ} \mathrm{C}$ (normal $36.0-37.0{ }^{\circ} \mathrm{C}$ ). The neck veins showed marginal engorgement. A lung examination revealed decreased, and coarsened breath sounds. The liver was palpable beneath the costal margin with a total vertical length of $14.4 \mathrm{~cm}$. In addition, pretibial edema was present. Chest computed tomography (CT) and contrast-enhanced CT angiography (CTA) revealed an anterior mediastinal mass with pericardial effusion and filling defects in the SVC and the left brachiocephalic vein (LBCV) (Figure 1). The mediastinal tumor did not appear to have any physical connection with the masses in 

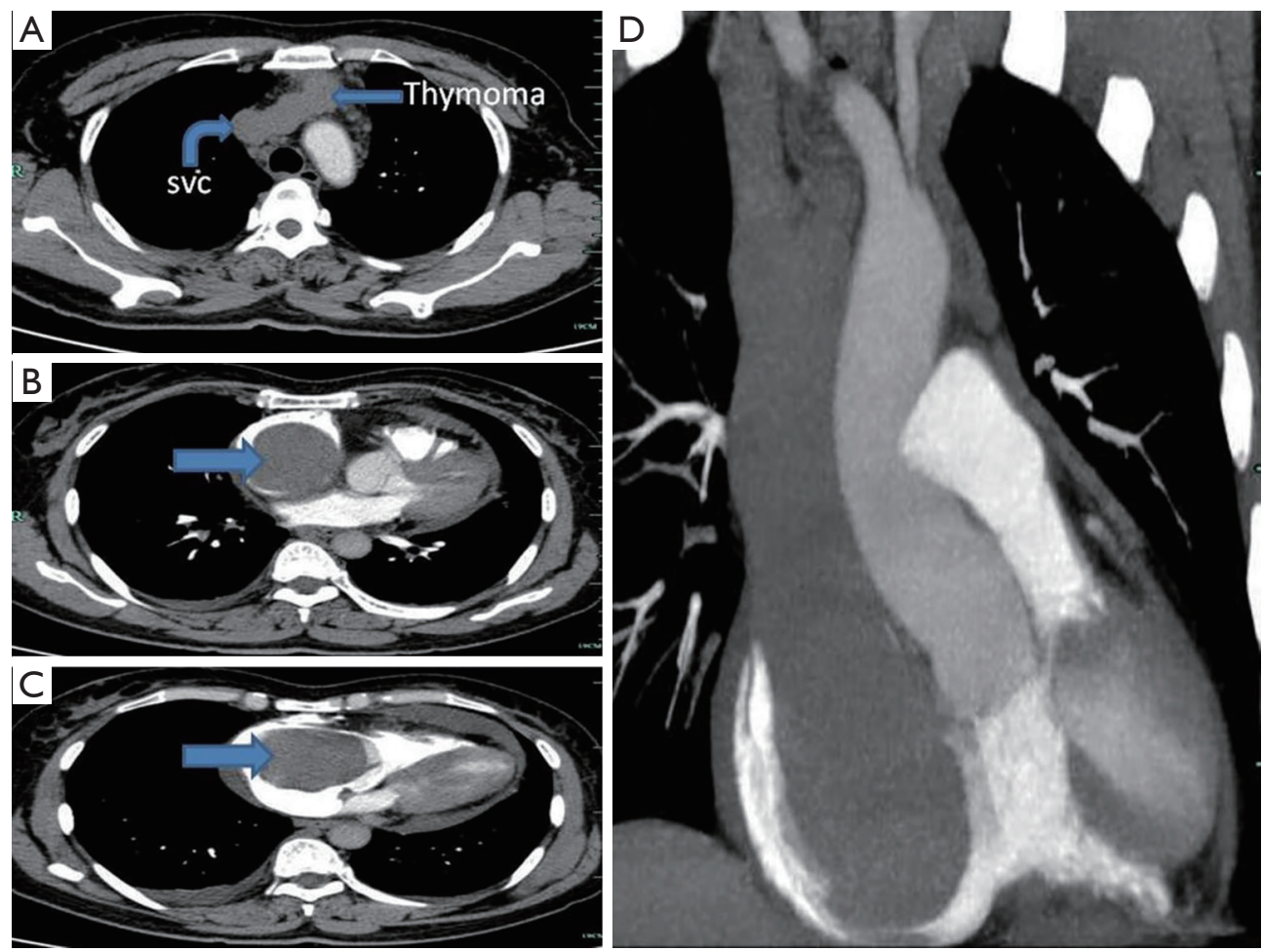

Figure 1 Pulmonary artery enhanced CT angiography (CTA) shows an anterior mediastinal mass and filling defects in the SVC and the LBV (A). The arrow indicate a giant neoplasm in the RA with pericardial effusion (B,C). Reconstruction in the sagittal plane, tumor in the SVC is extending to the RA, and blockage of the inlet of the tricuspid (D). SVC, superior vena cava; RA, right atrium; LBV, left brachiocephalic vein.

the SVC and LBCV according to the CT scan; therefore, metastasis of the tumor was considered. Echocardiography revealed moderate pericardial effusion with signs of cardiac tamponade and a large mass in the right atrium (RA) extending from the SVC. A cardiovascular ultrasound (CVUS) revealed a tumor in the SVC and the LBCV (size, $1.6 \mathrm{~cm} \times 1.2 \mathrm{~cm}$ ). Thus, the clinical tumor stage was designated Masaoka stage III (1981 system). The study was approved by the institutional ethics review board of The First Affiliated Hospital of Chongqing Medical University (The ID of ethics approval is 20183701). All patients provided written informed consent.

Prior to surgery, the patients internal jugular vein pressure was $32 \mathrm{cmH}_{2} \mathrm{O}$ (normal 5-12 $\mathrm{cm} \mathrm{H}_{2} \mathrm{O}$ ). The patient underwent a median sternotomy, at which time the sternocleidomastoid, pleural, and pericardial areas, along with the SVC and the LBCV, were observed to have been invaded by a mediastinal tumor (Figure $2 A$ ). The mediastinal portion of the tumor and the invaded organs were resected, and the LBCV was ligated. Next, the CPB was established by cannulating the ascending aorta and the inferior vena cava (IVC). The RA and the SVC were incised longitudinally. The tumors that were observed in the RA extended to the junction of the SVC and the LBCV and blocked the tricuspid valve inlet. In addition, there was a direct invasion of the wall of the SVC, the LBCV, and the IVC (Figure 2B). The complete tumor was resected, and the vessel wall was repaired using 4-0 Prolene sutures (Ethicon Inc., Somerville, NJ, USA). The main tumor in the RA, which extended to the SVC, measured $9 \times 8 \times 4.5 \mathrm{~cm}^{3}$. The tumor in the mediastinum measured $5 \times 5 \times 1.7 \mathrm{~cm}^{3}$. A portion of the remaining tissue was used for intraoperative frozen pathological examination and verified that there was no residual tumor in the remaining structures. Following surgery, the internal jugular vein pressure was $20 \mathrm{cmH}_{2} \mathrm{O}$. The patient was in the ICU of The First Affiliated Hospital of Chongqing Medical University for 3 days and discharged on the postoperative day 10. Echocardiography indicated that the postoperative tricuspid valve function returned to normal, and the SVC syndrome disappeared. The patient 

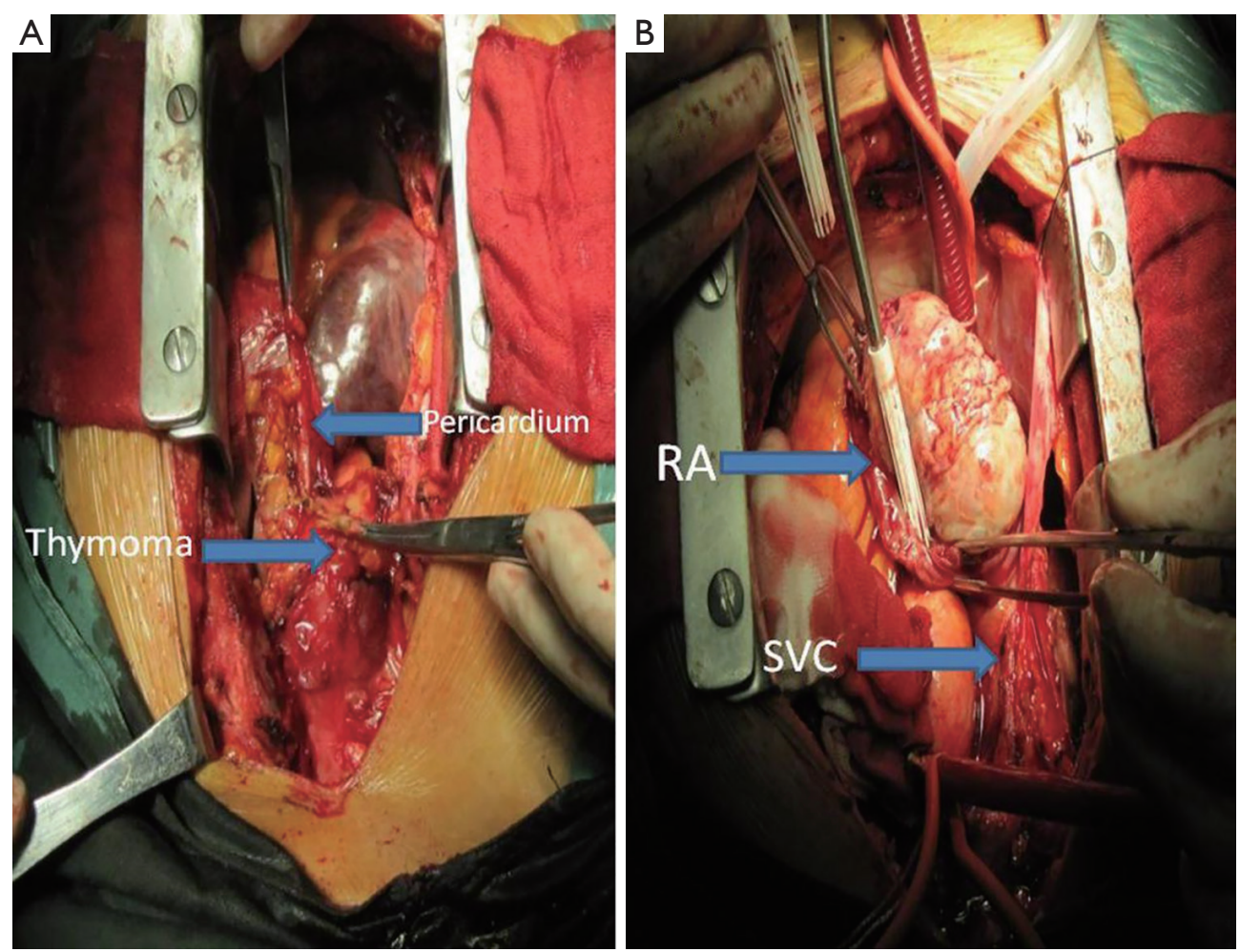

Figure 2 Photos of the operation. (A) A thymoma in the mediastinum invades the sternocleidomastoid, pleural area, and pericardial area; (B) a large thymoma in the RA extending to the SVC. SVC, superior vena cava; RA, right atrium.
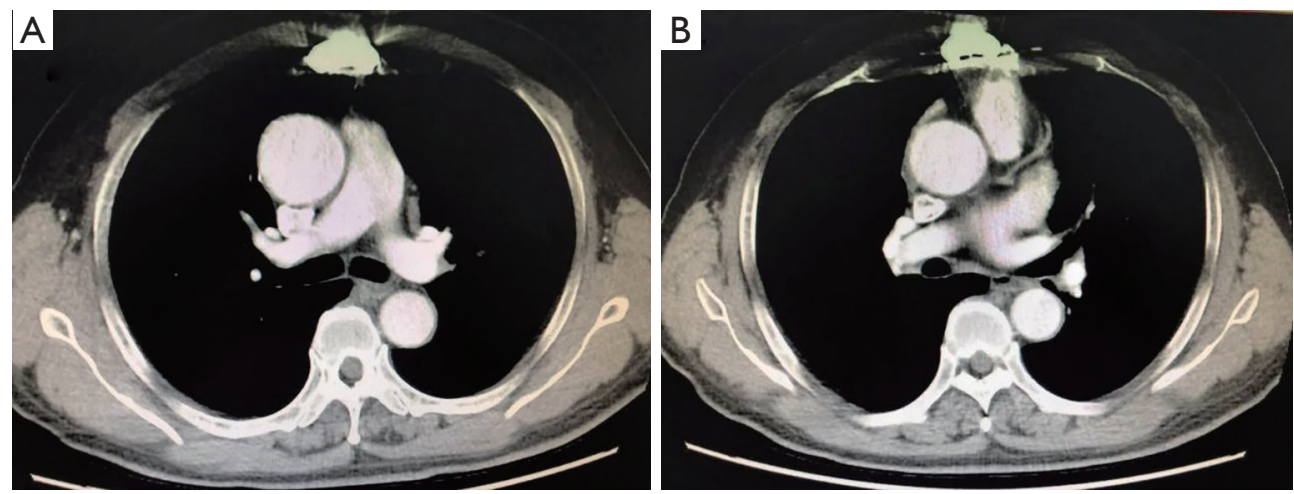

Figure 3 No residual tumor was observed upon a repeat CT scan at the six-month postoperative follow-up (A,B).

underwent radiotherapy $(61.6 \mathrm{~Gy} / 28 \mathrm{Fx})$ and chemotherapy including 2 cycles of GP plan (gemcitabine $1,500 \mathrm{mg}$ day $1+$ cisplatin $40 \mathrm{mg}$ day $1-3)$ and 1 cycle of NP plan (vinorelbine $40 \mathrm{mg}$ day $1+$ cisplatin $40 \mathrm{mg}$ day $1-3$ ). The patient has remained healthy as of the time of publication, and no residual tumor was observed upon a repeat CT scan at the six-month postoperative follow-up (Figure 3).

Pathological examination revealed the tumor to be a type
B3 thymoma (2004 WHO classification system; Figure 4). It had directly invaded the junction of the LBCV, from which the tumor thrombus extended into the SVC and the RA.

\section{Discussion}

Invasive thymoma with the intravascular invasion of the SVC extending into the RA has only rarely been reported. 


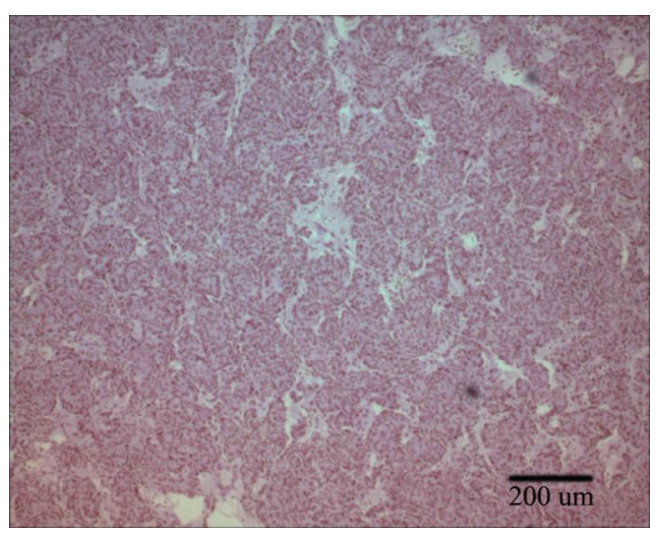

Figure 4 HE staining shows a mixed lymphocytic and epithelial cell type thymoma was categorized as a type B3 tumor under the WHO classification system.

Suzuki et al. first reported such a case in 1976 (4). However, since then, few cases have been reported worldwide. Kurata et al. reviewed 28 cases from 1976 to 2013 (5). It has been hypothesized that small thymomas in the anterior mediastinum infiltrate the brachiocephalic vein and grow along the venous stream into the SVC and down to the RA in a polypoid manner.

Treatment strategies for thymic carcinoma include surgery, chemotherapy, and radiotherapy. Shinohara et al. studied 56 thymic carcinoma patients who underwent surgery and found the overall rate of survival at 10 years to be $94.6 \%$ in patients with stage I or II disease and $77.1 \%$ in patients with stage III or IV disease (6). Yamazaki et al. examined 8 cases of invasive thymoma with the intravascular invasion of the SVC extending into the RA (7). All patients had initially undergone surgery, and 4 of these patients underwent chemotherapy, radiotherapy, or treatment via the two methods, and the average survival time was $>2$ years. The 3 patients who did not undergo chemotherapy or radiotherapy experienced an average survival time of $<1$ year. Therefore, multimodal therapy can be considered to be an effective treatment strategy. According to the Masaoka clinical staging system, the present patient was stage III. The clinical symptoms were serious, and quality of life was poor; therefore, surgical therapy was considered to be suitable. Following surgery, the internal jugular vein pressure had decreased to $12 \mathrm{cmH}_{2} \mathrm{O}$, and the swelling of the face and chest tightness had disappeared. The patient was referred for adjuvant chemotherapy and radiotherapy following discharge from hospital.

Performance of a CPB facilitates surgery and is relatively safe for the patient. Thus, CPB is often used in cardiac surgery. $\mathrm{CPB}$ requires systemic heparinization to destroy clotting material. Toker et al. reported a case that involved resection of an RA metastatic tumor via the transient cavapulmonary shunt (8). It has been recommended that CPB be avoided in cases of thoracic malignancy resection in order to decrease postoperative bleeding, thus resulting in a decrease in the number of transfusions required, the quantities of inotropic agents used, and the number of pleural effusions observed, potentially leading to early extubation. CPB was selected for surgery in the present case, as it was considered a mature and safe operative technique for cardiac surgery. The majority of the blood from the massive and continuous intraoperative bleeding was recycled, and an autotransfusion was performed. Following surgery, the total amount of bleeding tends to be $400 \mathrm{~mL}$. The patient did not receive a homologous transfusion, and the endotracheal intubation was extubated within 8 hours of surgery. The patient was in the ICU for 3 days and was discharged on postoperative day 10. Thus, CPB was considered to have been a suitable procedure during surgery.

\section{Conclusions}

Cardiopulmonary bypass is a safe method in the surgical treatment of invasive thymoma and can be wildly applied in clinical practice.

\section{Acknowledgments}

Funding: This study was supported by the Chongqing Natural Science Foundation (cstc2018jcyjAX0245).

\section{Footnote}

Conflicts of Interest: The authors have no conflicts of interest to declare.

Ethical Statement: The authors are accountable for all aspects of the work in ensuring that questions related to the accuracy or integrity of any part of the work are appropriately investigated and resolved. Written informed consent was obtained from the patient for publication of this manuscript and any accompanying images.

\section{References}

1. Zucali PA, De Pas T, Palmieri G, et al. Phase II Study 
of Everolimus in Patients With Thymoma and Thymic Carcinoma Previously Treated With Cisplatin-Based Chemotherapy. J Clin Oncol 2018;36:342-9.

2. Kneuertz PJ, Kamel MK, Stiles BM, et al. Robotic Thymectomy Is Feasible for Large Thymomas: A Propensity-Matched Comparison. Ann Thorac Surg 2017;104:1673-8.

3. Chick JFB, Osher ML, Cooper KJ, et al. Gianturco Z-Stent Fixation of a Modified Iliac Limb StentGraft Endoprosthesis for the Treatment of Malignant Superior Vena Cava Syndrome. Ann Vasc Surg 2017;45:263.e1-263.e4.

4. Suzuki Y, Dohi T, Tanaka S, et al. Surgical treatment in a case of malignant thymoma extending from the left brachiocephalic vein to the superior vena cava and the right atrium. Kyobu Geka 1976;29:357-61.

Cite this article as: Yang T, Hui R, Wu Q, Tian J, Chen H. An invasive thymoma extending into the superior vena cava and right atrium. Ann Transl Med 2019;7(18):498. doi: 10.21037/ atm.2019.08.59
5. Kurata A, Saji H, Ikeda N, et al. Intracaval and intracardiac extension of invasive thymoma complicated by superior and inferior vena cava syndrome. Pathol Int 2013;63:56-62.

6. Shinohara S, Hanagiri T, So T, et al. Results of surgical resection for patients with thymoma according to World Health Organization histology and Masaoka staging. Asian J Surg 2012;35:144-8.

7. Yamazaki K, Takeo S, Takeshita M, et al. Thymic carcinoma presenting as an intraluminal growth into the great vessels and the cardiac cavity. Ann Thorac Cardiovasc Surg 2002;8:163-6.

8. Toker A, Tireli E, Tanju S, et al. Transcaval invasion of right atrium by thymoma: resection via transient cava-pulmonary shunt. Eur J Cardiothorac Surg 2012;41:1175-7. 